\title{
Universality Classes of the Hwa-Kardar Model with Turbulent Advection
}

\author{
Nikolay V. Antonov ${ }^{1, \star}$, Nikolay M. Gulitskiy ${ }^{1, \star \star}$, Polina I. Kakin ${ }^{1, \star \star \star}$, and Vitaliy D. Serov ${ }^{1}$ \\ ${ }^{1}$ Department of Physics, Saint-Petersburg State University, 7/9 Universitetskaya naberezhnaya, \\ Saint Petersburg, 199034 Russia
}

\begin{abstract}
Self-organized critical system in turbulent fluid environment is studied with the renormalization group analysis. The system is modelled by the anisotropic stochastic differential equation for a coarse-grained field proposed by Hwa and Kardar [Phys. Rev. Lett. 62, 1813 (1989)]. The turbulent motion of the environment is described by the anisotropic $d$-dimensional velocity ensemble based on the one introduced by Avellaneda and Majda [Commun. Math. Phys. 131, 381 (1990)] and modified to include dependence on time (finite correlation time). Renormalization group analysis reveals three universality classes (types of critical behavior) differentiated by the parameters of the system.
\end{abstract}

\section{Introduction}

The phenomenon of self-organized criticality (SOC) is observed in numerous open nonequilibrium biological, ecological and social systems with dissipative transport [1,2]. While SOC is usually described by discrete models, the authors of [3] proposed a continuous anisotropic model, i.e., a differential stochastic equation for a smoothed (coarse-grained) field of the deviation from the average height of the sand profile. The model describes a running sandpile with a constant preferred transport direction.

This direction is determined by a unit constant vector $\boldsymbol{n}$, and the coordinate variable is decomposed into two components: $\boldsymbol{x}=\boldsymbol{x}_{\perp}+x_{\|} \boldsymbol{n}$ with the property $\left(\boldsymbol{x}_{\perp} \cdot \boldsymbol{n}\right)=0$. The stochastic equation for the height field $h(x)=h(t, \boldsymbol{x})$ is

$$
\partial_{t} h=v_{\perp 0} \partial_{\perp}^{2} h+v_{\| 0} \partial_{\|}^{2} h-\partial_{\|} h^{2} / 2+f
$$

where $h(x)$ is the deviation from the average, $\partial_{t}=\partial / \partial t, \partial_{\|}=(\boldsymbol{n} \cdot \partial), \partial_{\perp}=\partial / \partial x_{\perp}^{i}$ with $i=1, \ldots,(d-1)$, $d$ is the spatial dimension, $v_{\| 0}$ and $v_{\perp 0}$ are two diffusion coefficients, $f(x)$ is the Gaussian random noise with zero mean and a pair correlation function which reads

$$
\left\langle f(x) f\left(x^{\prime}\right)\right\rangle=D_{0} \delta\left(t-t^{\prime}\right) \delta^{(d)}\left(\boldsymbol{x}-\boldsymbol{x}^{\prime}\right) .
$$

The model (1) - (2) was discussed in relation with landscape erosion in [4-6]. It is well known that the turbulent motion of the environment can drastically change the critical behavior of the system;

\footnotetext{
^e-mail: n.antonov@spbu.ru

$\star \star$ e-mail: n.gulitskiy@spbu.ru

$\star \star \star$ e-mail: p.kakin@spbu.ru
} 
new non-equilibrium universality classes with unusual qualities can emerge [7-11]. This is the reason why we want to consider the critical behavior under the influence of the turbulent flow.

The replacement $\partial_{t} \rightarrow \nabla_{t}=\partial_{t}+v_{k} \partial_{k}$ introduces a coupling with the velocity field $\boldsymbol{v}(x)$. The latter is chosen in the form $\boldsymbol{v}(x)=v\left(t, \boldsymbol{x}_{\perp}\right) \boldsymbol{n}$. The model (1)-(2) with Gaussian $\delta$-correlated in time (rapid-changing) velocity ensemble has been studied in [12]. In this paper, we use a more realistic velocity field with finite correlation time. Its correlation function has the form

$$
\begin{gathered}
\left\langle v\left(t, \boldsymbol{x}_{\perp}\right) v\left(t^{\prime}, \boldsymbol{x}_{\perp}^{\prime}\right)\right\rangle=\int \frac{d \omega}{2 \pi} \int_{k_{\perp}>m} \frac{d \boldsymbol{k}}{(2 \pi)^{d}} e^{i \boldsymbol{k}\left(\boldsymbol{x}-\boldsymbol{x}^{\prime}\right)-i \omega\left(t-t^{\prime}\right)} B_{v}(\omega, \boldsymbol{k}), \text { where } \\
B_{v}(\omega, \boldsymbol{k})=2 \pi \delta\left(k_{\|}\right) \widetilde{B}_{v}(\omega, \boldsymbol{k}) \quad \text { and } \widetilde{B}_{v}(\omega, \boldsymbol{k})=B_{0} k_{\perp}^{5-d-(\xi+\eta)}\left(\omega^{2}+\left[\alpha_{0} v_{\perp 0} k_{\perp}^{2-\eta}\right]^{2}\right)^{-1}
\end{gathered}
$$

see [13-15]. Here the parameter $m$ provides infrared (IR) regularization, the parameter $B_{0}>0$ is a constant amplitude factor, while $\xi$ and $\eta$ are arbitrary exponents. The velocity correlator (3) involves two special cases: the limit $\alpha_{0} \rightarrow 0$ at fixed $B_{0} / \alpha_{0}$ corresponds to the case of the "frozen" velocity field that does not depend on time; the limit $\alpha_{0} \rightarrow \infty$ at fixed $B_{0} / \alpha_{0}^{2}$ corresponds to the ensemble with vanishing correlation time (rapid-change case). Since the model (1)-(2) has a preferred transport direction it is natural to choose an anisotropic ensemble for the turbulent environment.

The paper is organized as follows: the field theoretic formulation of the model is given in Sec. 2; Sec. 3 is devoted to the remormalization of the model while the fixed points of the renormalization group (RG) equation and corresponding universality classes are discussed in Sec. 4; Sec. 5 is reserved to a brief conclusion.

\section{Field theoretic formulation of the model}

The stochastic problem (1) - (3) is equivalent to the field theory of the set of fields $\Phi=\left\{h^{\prime}, h, v\right\}$ with the action functional

$$
\mathcal{S}(\Phi)=\frac{1}{2} h^{\prime} D_{0} h^{\prime}+h^{\prime}\left\{-\partial_{t} h-v \partial_{\|} h+v_{\perp 0} \partial_{\perp}^{2} h+v_{\| 0} \partial_{\|}^{2} h-\partial_{\|} h^{2} / 2\right\}+\mathcal{S}_{v} ;
$$

see, e.g., monographs $[16,17]$. The integrations over $x=\{t, \boldsymbol{x}\}$ and summations over the vector indices are implied; $h^{\prime}$ is the auxiliary (response) field. The term $\mathcal{S}_{v}$ appears from the Gaussian averaging over the velocity field $\boldsymbol{v}$ :

$$
\mathcal{S}_{v}=\frac{1}{2} \int d t \int d t^{\prime} \int d \boldsymbol{x}_{\perp} \int d \boldsymbol{x}_{\perp}^{\prime} v\left(t, \boldsymbol{x}_{\perp}\right) \widetilde{B}_{v}^{-1}\left(t-t^{\prime}, \boldsymbol{x}_{\perp}-\boldsymbol{x}_{\perp}^{\prime}\right) v\left(t^{\prime}, \boldsymbol{x}_{\perp}^{\prime}\right),
$$

where $\widetilde{B}_{v}^{-1}$ is the kernel of the linear operation $B_{v}^{-1}$.

The Feynman diagrammatic technique for the action (5) involves four nonzero bare propagators, namely $\langle v v\rangle_{0},\langle h h\rangle_{0},\left\langle h h^{\prime}\right\rangle_{0}$ and $\left\langle h^{\prime} h\right\rangle_{0}$, and two vertices related to the interaction terms $-h^{\prime} \partial_{\|} h^{2} / 2$ and $-h^{\prime}\left(v \partial_{\|}\right) h$. The coupling constants $g_{0}$ and $w_{0}$ are defined from canonical dimension analysis and are related to the parameters of the system as follows:

$$
D_{0}=g_{0}\left(v_{\|_{0}} v_{\perp_{0}}\right)^{3 / 2}, \quad B_{0}=w_{0} v_{\|_{0}} v_{\perp_{0}}^{2} .
$$

\section{Renormalization of the model}

The canonical dimensions analysis allows the determination of the ultraviolet (UV) divergences [16, 17]. The strongly anisotropic theories has usually three scales:

$$
[F] \sim[T]^{-d_{F}^{\omega}}\left[L_{\perp}\right]^{-d_{F}^{\perp}}\left[L_{\|}\right]^{-d_{F}^{\|}},
$$


where $T$ is the time scale and $L_{\perp}, L_{\|}$are two independent length scales in the corresponding subspaces.

The model (5) is logarithmic at $\varepsilon=4-d=0$ and $\xi, \eta=0$ when all the coupling constants are dimensionless. The UV divergence index of an arbitrary 1-irreducible Green function $\Gamma=\langle\Phi \cdots \Phi\rangle_{1 \text {-ir }}$ is given by the expression

$$
\delta_{\Gamma}=d+2-N_{h^{\prime}} d_{h^{\prime}}-N_{h} d_{h}-\left.N_{v} d_{v}\right|_{\varepsilon=\xi=\eta=0},
$$

where $N_{h}, N_{h^{\prime}}, N_{v}$ are the numbers of the corresponding fields in the function $\Gamma$, see, e.g. [17].

Galilean symmetry usually forbids some of the counterterms. Though the correlator (3) does not contain the Dirac function $\delta\left(t-t^{\prime}\right)$ necessary for Galilean symmetry, due to the anisotropy of the model and the incompressibility of the velocity field, the action (5) is, nevertheless, invariant under the following Galilean transformations:

$$
h(t, \boldsymbol{x}) \rightarrow h(t, \boldsymbol{x}+t \boldsymbol{u}), \quad h^{\prime}(t, \boldsymbol{x}) \rightarrow h^{\prime}(t, \boldsymbol{x}+t \boldsymbol{u}), \quad \boldsymbol{v}(t, \boldsymbol{x}) \rightarrow \boldsymbol{v}(t, \boldsymbol{x}+t \boldsymbol{u})-\boldsymbol{u},
$$

where $\boldsymbol{u}=u \boldsymbol{n}$, see [14] for more details. Eqs. (9) means that the scalar velocity changes as $v\left(t, \boldsymbol{x}_{\perp}\right) \rightarrow$ $v\left(t, \boldsymbol{x}_{\perp}\right)-u$ and $\boldsymbol{x}_{\perp}$ remains unchanged in all of the fields in (9).

The analysis of the possible divergent functions shows that the theory (5) is multiplicatively renormalizable with only one nontrivial renormalization constant, $Z_{\nu_{\|}}$:

$$
\mathcal{S}_{R}(\Phi)=\frac{1}{2} h^{\prime} D h^{\prime}+h^{\prime}\left\{-\partial_{t} h-v \partial_{\|} h+v_{\perp} \partial_{\perp}^{2} h-\partial_{\|} h^{2} / 2+Z_{v_{\|}} v_{\|} \partial_{\|}^{2} h\right\}+\mathcal{S}_{v}
$$

In the leading order of the triple expansion in $\varepsilon, \xi$, and $\eta$ (note, that we use the minimal subtraction scheme), this constant reads

$$
Z_{\nu_{\|}}=1-\frac{w}{2 \alpha(1+\alpha) \xi}-\frac{3 g}{16 \varepsilon},
$$

where $g, w$, and $\alpha$ are renormalized counterparts for the bare coupling constants; moreover, we assumed that the constants $g$ and $w$ absorbed the coefficient $S_{d} /(2 \pi)^{d}$ with $S_{d}$ being the area of the unit sphere in the $d$-dimensional space.

\section{Fixed points and scaling regimes}

It is well known that at large-distances, the long-time behavior of a renormalized model is governed by the IR attractive fixed points the coordinates of which are found from the conditions

$$
\beta_{g}\left(g^{*}, w^{*}, \alpha^{*}\right)=\beta_{w}\left(g^{*}, w^{*} \alpha^{*}\right)=\beta_{\alpha}\left(g^{*}, w^{*}, \alpha^{*}\right)=0
$$

together with the restriction that the matrix $\Omega_{i j}=\partial \beta_{i} /\left.\partial g_{j}\right|_{g^{*}, w^{*}, \alpha^{*}}$ should be positive definite. The symbol $\beta_{q}$ in Eqs. (12) denotes so-called $\beta$-functions of the coupling constants $q[16,17]$.

Direct analysis of Eqs. (12) shows that there are two groups of fixed points in our model. The first group has the coordinate $\alpha^{*}=0$ and contains three different points: the point (a) with two other coordinates $w^{\prime *} \equiv w / \alpha=0, g^{*}=0$ which is IR attractive at $\varepsilon, \eta, \xi<0$; the point (b) with coordinates $w^{\prime *}=2 \xi, g^{*}=0$ which is IR attractive at $\eta<0, \xi>0, \varepsilon<3 \xi / 2$; and the point (c) with coordinates $w^{\prime *}=0, g^{*}=32 \varepsilon / 9$ which is IR attractive at $\eta<0, \varepsilon>0, \xi<2 \varepsilon / 3$. From these expressions it follows that the nonlinear term in Eq. (1) and the turbulent motion of the environment are IR irrelevant for the points (b) and (c), respectively.

The second group has the coordinate $\alpha^{*}=\infty$ and consists of the same set of points as above, with the replacements $w^{\prime} \rightarrow w^{\prime \prime} \equiv w / \alpha^{2}, \eta \rightarrow-\eta$ and $\xi \rightarrow \xi-\eta$. 
The presence of IR attractive fixed points in the model (5) leads to scaling behavior of measurable quantities with different critical dimensions which depend on the values of $\eta, \varepsilon$ and $\xi$. The Kolmogorov values of the exponents $\xi=8 / 3$ and $\eta=4 / 3$ lie either in the stability region of the fixed point (b) of the second group (universality class of the rapid change ensemble) or the fixed point (c) of the same second group (universality class of the Hwa-Kardar model) depending on whether $\varepsilon$ is smaller or larger than 2 , respectively.

\section{Conclusion}

In this paper the model of self organized criticality under the influence of a turbulent motion of the environment was studied using the field theoretic approach. The coordinates of the fixed points of the RG equation were found in the one-loop approximation, that is, in the leading order of the triple expansion in $\varepsilon, \xi$, and $\eta$. The presence of the set of IR attractive fixed points leads to scaling behavior of measurable quantities; the corresponding critical dimensions depend on the type of a fixed point, i.e., on the values of $\eta, \varepsilon$ and $\xi$.

It turns out that the velocity field has either infinite or vanishing correlation time in the IR limit. For either case the IR behaviour of the model is divided into three universality classes: the trivial regime (ordinary diffusion), the regime of the pure velocity field (nonlinearity of the Hwa-Kardar equation is IR irrelevant), and the regime of the model without advection. The regime where both the nonlinearity of the initial equation and the turbulent advection are important appears to be prohibited.

\section{Acknowledgements}

The authors thank the Organizers of the conference "Mathematical Modelling and Computational Physics 2019" for the possibility to present the results reported in the paper. The reported study was funded by RFBR according to the research project No. 18-32-00238. N.M.G. acknowledges the support from Saint Petersburg State University within the travel grant No. 40219299.

\section{References}

[1] P. Bak, C. Tang, K. Wiesenfeld, Phys. Rev. Lett. 59, 381 (1987)

[2] P. Bak, How Nature Works: The Science of Self-Organized Criticality (Copernicus, N.Y., 1996)

[3] T. Hwa, M. Kardar, Phys. Rev. A 45, 7002 (1992)

[4] B. Tadić, Phys. Rev. E 58, 168 (1998)

[5] R. Pastor-Satorras, D.H. Rothman, Phys. Rev. Lett. 80, 4349 (1998)

[6] R. Pastor-Satorras, D.H. Rothman, J. Stat. Phys.93, 477 (1998)

[7] A. Onuki, K. Yamazaki, K. Kawasaki, Ann. Phys. 131, 217 (1981)

[8] D. Beysens, M. Gbadamassi, L. Boyer, Phys. Rev. Lett 43, 1253 (1979)

[9] R. Ruiz, D.R. Nelson, Phys. Rev. A 23, 3224 (1981)

[10] N.V. Antonov, A.A. Ignatieva, J. Phys. A: Math. Gen. 39, 13593 (2006)

[11] N.V. Antonov, A.S. Kapustin, A.V. Malyshev, Teor. Math. Phys. 169, 1470 (2011)

[12] N.V. Antonov, P.I. Kakin, EPJ Web of Conferences 108, 02009 (2016)

[13] M. Avellaneda, A. Majda, Commun. Math. Phys. 131, 381 (1990)

[14] N.V. Antonov, A.V. Malyshev, J. Stat. Phys. 146, 33 (2012)

[15] N.V. Antonov, N.M. Gulitskiy, Phys. Rev. E 92, 043018 (2015)

[16] J. Zinn-Justin Quantum Field Theory and Critical Phenomena (Clarendon Press, Oxford, 1989)

[17] A.N. Vasiliev The Field Theoretic Renormalization Group in Critical Behavior Theory and Stochastic Dynamics (Chapman \& Hall/CRC, Boca Raton, 2004) 